\title{
A DC Electric Panel Remote Lab
}

\author{
http://dx.doi.org/10.3991/ijoe.v12i04.5096
J.B. Silva ${ }^{1}$, J.P.S. Simão ${ }^{1}$, M.A.S. Cristiano ${ }^{1}$, P.C. Nicolete ${ }^{1}$, C. Heck ${ }^{1}$, K.S. Coelho ${ }^{2}$
${ }^{1}$ Federal University of Santa Catarina, Araranguá, Brazil \\ ${ }^{2}$ E.E.B. Apolônio Ireno Cardoso, Araranguá, Brazil
}

\begin{abstract}
The remote experimentation labs provide students an opportunity to conduct scientific experiments at any time and on any device connected to the Internet. To this end, each experiment must be designed to meet these characteristics and educational needs. In this paper it will be described the implementation of the "DC Electrical Panel", addressing its main features such as modules, architecture, modeling, and other technical aspects that allow the remote access. Currently the Remote Experimentation Laboratory (RExLab) has eight (8) labs in the areas of physics and biology, in the three levels of education; Elementary School, Secondary School and Higher Education.
\end{abstract}

Index Terms - e-learning, physics, remote labs, STEM.

\section{INTRODUCTION}

Remote labs are devices that allow students to work with real lab devices through the Internet, using conventional devices (desktop, laptop, etc.) as well as mobile devices (tablets, smartphones, etc.) [1].

The "DC Electric Panel" lab aims to help high school and higher education students to make practices related to resistors association (serial, parallel and mixed), as well as the electronic circuit analysis into direct current using the basic laws of circuits analysis: Ohm's Law and Kirchoff's Circuit Laws, from the use of a practical approach to implementing these laws.

In the overview of the proposed service (Figure 1), the block "MRE" includes the remote labs, services and applications for access support. This block is responsible for the controlling and observation of remote experiments, in addition to the access to educational content. The "Educational Content" includes all the background material for the use of the experiment: manuals, handout.

For the management of educational content, in the block "VLE" (Virtual Learning Environment), it is used Moodle (Modular Object-Oriented Dynamic Learning Environment) because of its flexibility.

\section{ARCHITECTURE}

The construction of remote labs is based on open hardware and software in order to encourage the replication of the project and integrate these technologies in a distributed environment for teaching and learning. All experiments are implemented from the standardized architecture of hardware and basic software. Some elements such as sensors and actuators vary according to the specificities of the lab.

The central device on the DC Electric Panel lab is an Embedded Computer (EC). The Raspberry Pi B+ is used, whose main function is to mediate the access to other hardware devices through the network.
The Raspberry Pi is a small computer based on a system on a chip (SoC) Broadcom BCM2835, which includes a ARM1176JZFS processor running at $700 \mathrm{MHz}$, VideoCore IV GPU, and $512 \mathrm{MB}$ of RAM in its latest revision. The Raspberry IP was developed in the UK by the Raspberry Pi Foundation [2]

The function of the "Embedded Computer" is to provide interfacing and management for the connection between the network (web) and "Acquisition and Control Board" (ACB). The EC accesses the ACB to collect the sensor data or to send commands to the actuators, where the communication is done via UART (Universal Asynchronous Receiver / Transmitter) that communicates via MODBUS2 protocol.

Modbus is a data communication protocol used in industrial automation systems. It is one of the most used protocols in networks Programmable Logic Controllers (PLC) for acquisition of signals ( 0 or 1 ) instruments and control actuators.

The "Acquisition and Control Board" (Figure 2) aims to control the sensors and actuators of the lab. This block is based on the ARM Cortex-M3 PLC1752 processor running at $80 \mathrm{MHz}$ clock frequency. The choice of this ARM model is due to its availability and cost/benefit.

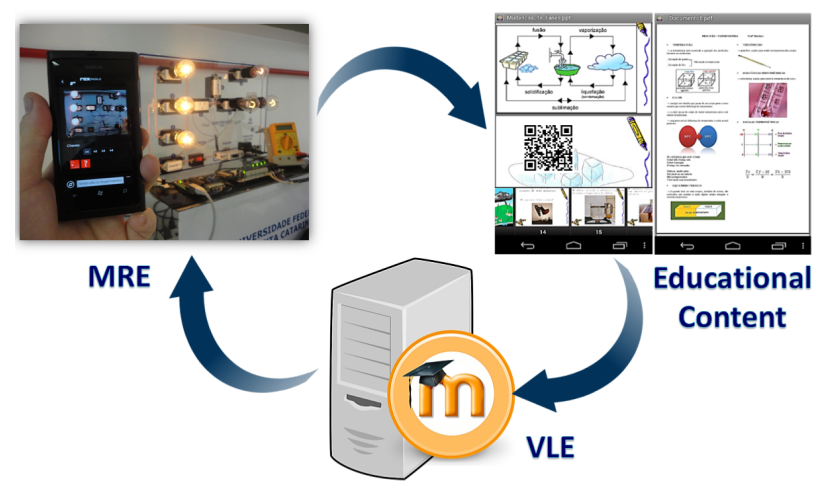

Figure 1. Service overview

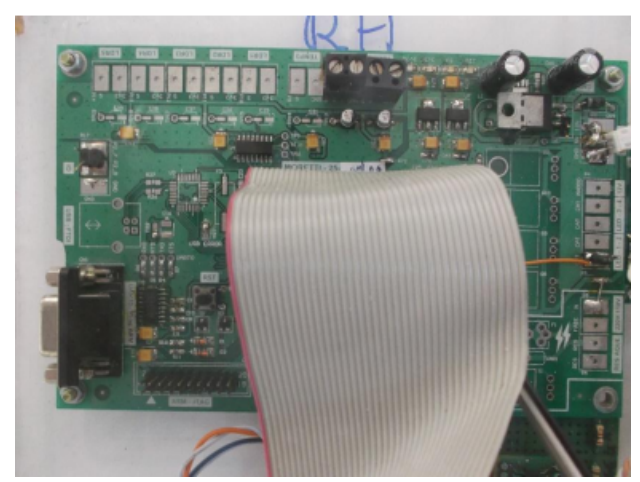

Figure 2. Acquisition and Control Board 
The LPC1752 is a microcontroller based on ARM Cortex-M3 architecture that operates at frequencies up to 100 $\mathrm{MHz}$. This type of microcontroller is designed for embedded applications since they are characterized by a high level of integration and low power consumption. This microcontroller incorporates a pipeline of three stages and has data buses and separate statements, inspired by the Harvard architecture.

The LPC1752 also includes 64KB flash memory, data memory 16KB (SRAM) 53 pin I / O, USB, UART (4), $\mathrm{I}^{2} \mathrm{C}$ (3), SPI (2), and ADC (6) Timers (6) and PWM (6). It operates with $3.3 \mathrm{~V}$ voltage.

The "Acquisition and Control Board" acts as a manager that controls all actuators and read all sensors of the experiments. It also stores all the updated sensor data to be accessed by external physical devices. Also it is on standby, waiting for commands to drive the actuators. The implementation of the ACB is sought to apply a certain degree of autonomy from the sensors and actuators.

\section{DC ELECTRIC PANEL}

The Electric Panel DC lab consists of a panel composed of seven (7) resistors, seven (7) switches, LEDs and a power supply. In this lab it is possible to obtain voltage and current readings in various parts of circuit according to the different combinations of keys inserted in the electrical circuit.

The resistors used in the lab are constructed from carbon film, $3 \mathrm{~W}$ power and $5 \%$ tolerance. The use of this power is due to its physical size, in order to provide viewing even through the webcam installed to monitor the experiment.

The experiment circuit includes seven switches that are controlled by the user in order to obtain the desired configuration for a given circuit. The keys on the panel are represented by six green LEDs (Light Emitting Diode). When the switch (relay) is not triggered, it will not circulate the electric current at that stage of the circuit and the LEDs will not display brightness. When pressing the key, the available for the user interface, the key will be electronically actuated, closing the circuit and the LEDs will light up .

In some points of the circuit are implemented LEDs bar, in order, to enhance the voltage drop at certain points. So, the more LEDs will be linked more tension, and vice versa, operation similar to VU meter (Volume Unit) of LEDs of stereos.

The project "Electrical Panel DC" was made using modern techniques of modeling and development of circuits. The Figure 5 shows the modeling of electronic design.

In order to enrich the practical activities, the experiment is equipped with ammeters and voltmeters, the actual construction, which return "feedback" of the readings at the points indicated to users.

Similarly to ammeters and voltmeters circuits and boards for the implementation of resistors, relays and LEDs bars were also designed and constructed exclusively for the remote experiment.

\section{A. Voltmeters and Ammeters Operation}

These devices are based on the ARM Cortex-M3 LPC1752 processor. The multimeter or ammeter function

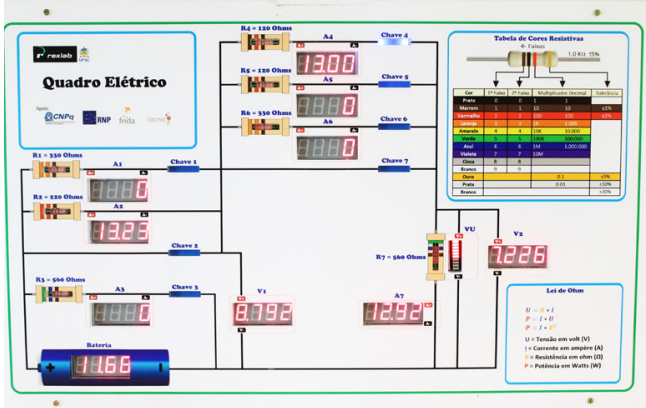

Figure 3. Electric Panel DC Lab

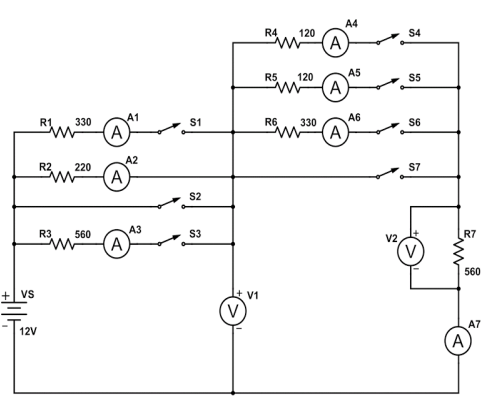

Figure 4. Electric diagram

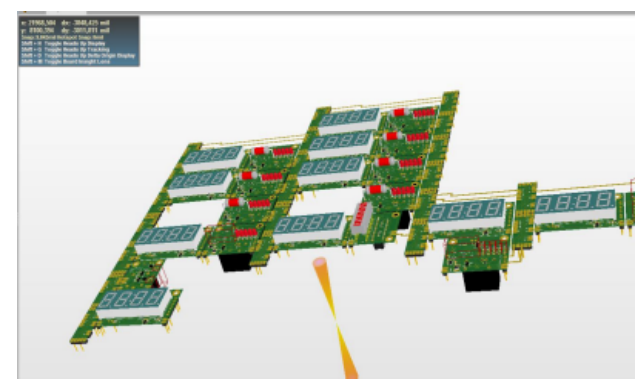

Figure 5. Electronic modeling

is selected into the device through a jumper, which selects what to display on display 7 (seven) segments.

For current reading it was used an analog current sensor INA-169, suitable for measuring low DC current values in this case is dimensioned for reading $50 \mathrm{~mA}$ maximum current.

The operation of this integrated circuit basically consists in converting from current to voltage reading "voltage drop" in resistor "shunt", where this loss is related to the current passing through it. The shunt resistor is nothing more than a low-value resistor connected in series with the load used to provide feedback to the microcontroller, thus the microcontroller identifies whether the current supplied complies (or not) as expected.

The INA-169 proportionally converts the input voltage into a small output current. In INA 169-current output is converted back to voltage through an external resistor (RL) connected to the GND (Ground) of the circuit. This is useful to measure the line current through an ADC (Analog Digital Conversion) or other devices.

Reading of tensions in the circuit was implemented a resistive divider capable of reading voltages from 0 to $15 \mathrm{~V}$ with a microcontroller $(\mu \mathrm{C})$ of $3 \mathrm{v} 3$.

The measurement must be in common mode, i.e., both the INA-169 as the measurement unit (cargo or load) should have a common ground. The load supply voltage 
and the NAC-169 from the supply voltage can be $60 \mathrm{~V}$ between the $2 \mathrm{v} 7$ and are mutually independent.

The differential voltage at the shunt resistor must be a maximum of $0.5 \mathrm{~V}$, which produces a current of $500 \mu \mathrm{A}$ but voltages above that do not damage integrated circuit (IC), although saturate the output voltage. The differential measurement must be unipolar, where the positive horny must be connected to positive input of IC, if there is a reversal, the produced current is zero.

Therefore, the INA-169 only measures DC current, that because the input voltage on Vin + should always be greater than Vin- input. The shunt resistor (Rs) should be placed as close as possible to the IC terminals in order to decrease the maximum resistance in series with the Rs produced by track.

The value of Rs depends on the application relating to accuracy and the voltage loss in the measurement line. Rs with higher values are more accurate at low currents, but at high currents there is much loss of pressure and a lot of power is dissipated in this case you must use rs with very low values.

Generally, value for Rs is used to provide a full range where the voltage difference varies in the range from $50 \mathrm{mV}$ to $100 \mathrm{mV}$. Values above $500 \mathrm{mV}$ the ina 169 saturates and values below $35 \mathrm{mV}$ the ina169 starts measuring the wrong way. Usually are used Rs values of $0.1,1$ and $10 \mathrm{ohms}$.

The value of $\mathrm{R} 1$ is selected to a full scale output voltage, as the output impedance of the INA-169 is high and we can use a resistor with a value of up to $100 \mathrm{~K}$ with good measuring accuracy. However, the addition circuit as an ADC, you must have your input impedance greater than $\mathrm{R} 1$ if it is not, you must use an operational amplifier configured as buffer.

\section{B. Access, manipulation and feedback}

When accessing the experiment, a page containing a description of the experiment is shown; however, in order to ensure exclusive access to the resource, if another person is using the lab, the user receives a message containing how many people are waiting before him, and how long before he or she can access the lab.

The experiment page (Figure 6) is formed by the video streaming, the controls, electric diagram, and the readings from the sensors. The block "What?" explains what is happening, and the block "How?" explains how the user controls the experiment.

When the user clicks on the control toggle selectors, a command call is sent to the lab API, connecting the relay that triggers the switch. The process can be seen through the images from the streaming.

The user can also generate a report of the experiment, consisting of a .pdf document containing user data (when logged in the system), date and time, the results of sensor readings and resistors, and a camera screenshot.

The system saves the logs information about the user lab accesses, including user, location, time (session length), operating system and browser. This information is used for analytics purposes.

The lab described here, as well as all other experiment provided by RExLab, aims to develop and deploy a platform that integrates virtual environment for teaching and
DC Eletric Panel
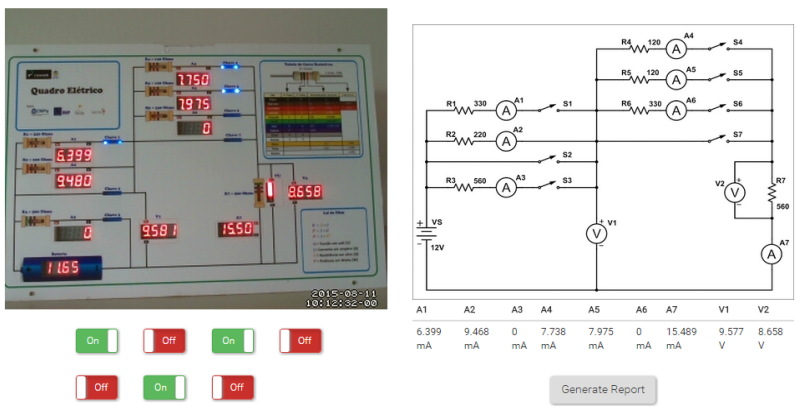

Figure 6. Lab access interface

\section{CONCLUSION}

learning with remote experiments. This platform is also available online open educational content that can be accessed by mobile or conventional devices.

Each experiment is associated with a technical manual and a didactic manual, offering tools for developing practice in the classroom. Objective with this vocational awakening students' interest in STEM areas (Science, Technology, Engineering, and Mathematics) and scientific and technological research, and contribute and stimulate the integration of technology in basic education in Brazilian public schools.

This lab is a prototype, and is being validated by high school physics teachers in their classes in Araranguá, Brazil.

\section{REFERENCES}

[1] J. de Lima, W. Rochadel, A. Silva, J. Simao, J. da Silva, and J. Alves, "Application of remote experiments in basic education through mobile devices," in Global Engineering Education Conference (EDUCON), 2014 IEEE, 2014, pp. 1093-1096. http://dx.doi.org/10.1109/educon.2014.6826245

[2] RASPBERRYPI. (2015). Raspberry Pi. Available at https://www.raspberrypi.org/.

\section{AUTHORS}

J.B. Silva is a professor in the Graduate Program in ICT (PPGTIC), at the Federal University of Santa Catarina (juarez.silva@ieee.org).

J.P.S. Simão is an ICT undergraduate student at the Federal University of Santa Catarina (jpsimao@grad.ufsc.br).

M.A.S. Cristiano. is a doctoral student in the Graduate Program in Knowledge Engineering and Management (PPEGC), at the Federal University of Santa Catarina (marta.php@gmail.com).

P.C. Nicolete is a master's student in the Graduate Program in ICT (PPGTIC), at the Federal University of Santa Catarina (priscila.cadorin@posgrad.ufsc.br).

C. Heck is a master's student in the Graduate Program in ICT (PPGTIC), at the Federal University of Santa Catarina (carine.heck@posgrad.ufsc,br).

K.S. Coelho is master in Scientific and Technological Education, and a physics teacher at the E.E.B. Apolônio Ireno Cardoso (kakascoelho@hotmail.com).

Submitted 27 September 2015. Published as resubmitted by the authors 23 November 2015. 